\title{
Desempenho de macieiras 'Imperial Gala' e 'Mishima Fuji' em diferentes porta-enxertos
}

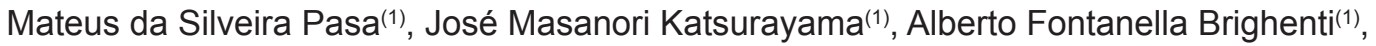 \\ Jerônimo Vieira de Araújo Filho ${ }^{(1)}$ e José Itamar da Silva Boneti ${ }^{(1)}$
}

\begin{abstract}
(1)Empresa de Pesquisa Agropecuária e Extensão Rural de Santa Catarina, Estação Experimental de São Joaquim, Rua João Araújo Lima, no 102, Bairro Jardim Caiçara, CEP 88600-000 São Joaquim, SC, Brasil. E-mail: mateuspasa@epagri.sc.gov.br, masanori@epagri.sc.gov.br, albertobrighenti@epagri.sc.gov.br, jeronimofilho@epagri.sc.gov.br, fito.boneti@gmail.com
\end{abstract}

Resumo - O objetivo deste trabalho foi avaliar a influência de diferentes porta-enxertos no desempenho das cultivares de macieira (Malus domestica) Imperial Gala e Mishima Fuji. O delineamento experimental utilizado foi o de blocos ao acaso, com quatro repetições. Utilizaram-se os porta-enxertos CG.969, CG.874, CG.210, CG.56, CG.008, JM.2 e JM.7, além da combinação de Marubakaido com interenxerto de M.9 (Marubakaido/M.9). Avaliaram-se as variáveis: produção por planta, produtividade, número de frutos por planta, massa de fruto, área da seção transversal do tronco, sólidos solúveis, firmeza do fruto e índice iodo-amido. A produtividade de ambas as cultivares foi maior com os porta-enxertos Marubakaido/M.9, JM.2, CG.008, CG.874, CG.210 e CG.56. Além disso, os porta-enxertos CG.008, CG.874, CG.210 e CG.56 reduzem o vigor da cultivar-copa, mas sem reduzir a produtividade. O teor de sólidos solúveis dos frutos é maior quando se utilizam porta-enxertos menos vigorosos.

Termos para indexação: Malus domestica, controle de vigor, produtividade, qualidade de fruto, série CG, série JM.

\section{Performance of 'Imperial Gala' and 'Mishima Fuji' apples on different rootstocks}

\begin{abstract}
The objective of this work was to evaluate rootstock effect on the performance of the Imperial Gala and Mishima Fuji apple (Malus domestica) cultivars. The experimental design was a randomized complete block with four replicates. The CG.969, CG.874, CG.210, CG.56, CG.008, JM.2, and JM.7 rootstocks were used, besides the combination of Marubakaido with the M.9 interstock (Marubakaido/M.9). The assessed parameters were: production per tree, yield, number of fruit per tree, fruit weight, trunk cross-sectional area, soluble solids, fruit firmness, and starch-iodine index. Both cultivars showed greater yield when grafted onto the Marubakaido/M.9, JM.2, CG.008, CG.874, CG.210, and CG.56 rootstocks. In addition, the CG.008, CG.874, CG.210, and CG.56 rootstocks decrease tree vigor, but without reducing yield. Soluble solids content in fruit is greater when less vigorous rootstocks are used.
\end{abstract}

Index terms: Malus domestica, vigor control, yield, fruit quality, CG series, JM series.

\section{Introdução}

A maçã (Malus domestica Borkh) é a segunda fruta de clima temperado mais produzida no Brasil, tendo atingido uma produção de 1.378 .617 toneladas. Os Estados do Rio Grande do Sul e Santa Catarina são responsáveis por cerca de $97 \%$ da produção brasileira (Instituto Brasileiro de Geografia e Estatística, 2014). Vários fatores foram responsáveis pelo grande desenvolvimento da cultura da macieira no País, com destaque para a definição de cultivares, como Gala e Fuji, e de seus clones, capazes de atender às exigências dos consumidores (Fachinello et al., 2011). No entanto, embora haja pomares altamente produtivos, a produtividade média ainda é baixa $\sim 37$ $\mathrm{Mg} \mathrm{ha}^{-1}$ (Instituto Brasileiro de Geografia e Estatística, 2014). Esse cenário é atribuído, em parte, às baixas densidades de plantio adotadas e à utilização de porta-enxertos vigorosos.

Para superar essa situação, sistemas mais eficientes e rentáveis, como plantios em alta densidade, podem ser utilizados. As principais vantagens do adensamento de pomares são a precocidade de entrada em produção, a alta produtividade, a alta qualidade de frutos e o menor custo com mão de obra (Hampson et al., 2002). Assim, o aumento da densidade de plantio é um dos fatores mais importantes no aumento da produtividade (Petri et al., 2011) e da rentabilidade (Kreuz et al., 2006) de 
pomares de macieira, os quais dependem da utilização de técnicas de manejo, como a correta escolha do porta-enxerto, para controlar o tamanho das plantas (Pasa \& Einhorn, 2014). Para isso, as limitações e as vantagens de cada porta-enxerto devem ser avaliadas para a escolha daqueles que apresentam as melhores características para cada condição (Gjamovski \& Kiprijanovski, 2011).

De acordo com Wertheim (2002), além da influência do porta-enxerto, o vigor das plantas enxertadas também é determinado pelas condições de solo, clima e cultivar-copa. O município de São Joaquim, responsável por aproximadamente $38 \%$ do total de maçãs produzido em Santa Catarina (Síntese..., 2013), apresenta condições de clima e solo peculiares, com predominância de solos rasos, pedregosos e com afloramento de rochas. Atualmente, o porta-enxerto Marubakaido, em razão do seu elevado vigor, ainda é o mais utilizado (Denardi et al., 2015), apesar de em média densidade de plantio, com aproximadamente 1.000 plantas por hectare. A combinação desse porta-enxerto com interenxerto (filtro) do ananizante M.9 tem possibilitado maior adensamento dos pomares. Contudo, essa combinação apresenta algumas desvantagens, como incidência de pulgão-lanígero no interenxerto suscetível (M.9), maior custo das mudas e desuniformidade do pomar. Esta última é resultante da dificuldade de padronizar a altura de enxertia e o tamanho do interenxerto, que são duas variáveis que comprovadamente alteram o vigor da cultivar-copa (Di Vaio et al., 2009). Além disso, as plantas tornam-se muito vigorosas à medida que envelhecem (Robinson, 2011), o que dificulta o manejo. Logo, é fundamental que se identifiquem porta-enxertos alternativos aos tradicionalmente utilizados.

Dos porta-enxertos recentemente desenvolvidos no mundo, os da série americana Geneva são os mais completos em termos de características agronômicas requeridas para uso no Brasil (Denardi et al., 2015). Entre essas características, destacam-se: resistência ao pulgão-lanígero, melhor ramificação e ângulo de abertura dos ramos, incremento de produtividade e tolerância à doença do replantio (Fazio et al., 2013). Além desses porta-enxertos, os da série japonesa JM apresentam resistência a algumas doenças e pragas importantes, bem como controle de vigor e facilidade de propagação (Soejima et al., 1998), o que os torna potenciais para uso nas condições da região de São Joaquim, SC.

O objetivo deste trabalho foi avaliar a influência de diferentes porta-enxertos no desempenho das cultivares de macieira (Malus domestica) Imperial Gala e Mishima Fuji.

\section{Material e Métodos}

O trabalho foi conduzido na Estação Experimental de São Joaquim da Empresa de Pesquisa Agropecuária e Extensão Rural de Santa Catarina, localizada em São Joaquim, SC ( $28^{\circ} 17^{\prime} 39^{\prime \prime} \mathrm{S}, 49^{\circ} 55^{\prime} 56^{\prime \prime} \mathrm{W}$, a $1.415 \mathrm{~m}$ de altitude), entre 2010 e 2015 . O clima, de acordo com a classificação de Köppen-Geiger, é do tipo $\mathrm{Cfb}$, mesotérmico úmido, sem estação seca e com verão fresco (Benez, 2005). O acúmulo médio de temperaturas iguais ou inferiores a $7,2^{\circ} \mathrm{C}$ na região é de 900 horas. As condições meteorológicas durante a condução do experimento estão descritas na Figura 1. O solo do campo experimental é classificado como Cambissolo Húmico, de acordo com o Sistema Brasileiro de Classificação de Solos (Santos et al., 2013). As características químicas e o teor de argila do solo da área experimental, no momento da implantação do experimento, foram: $5,5 \mathrm{de} \mathrm{pH}$ (em $\left.\mathrm{H}_{2} \mathrm{O}\right)(1: 1)$; índice SMP de 5,0; 7,1 $\mathrm{mg} \mathrm{L}^{-1}$ de fósforo; $154 \mathrm{mg} \mathrm{L}^{-1}$ de potássio; $6,3 \%$ de matéria orgânica $(\mathrm{m} / \mathrm{v}) ; 0,0 \mathrm{cmol} \mathrm{L}^{-1}$ de alumínio; 7,3 $\mathrm{cmol} \mathrm{L}^{-1}$ de cálcio; $3,4 \mathrm{cmol} \mathrm{L}^{-1}$ de magnésio; e $32 \%$ de argila. A análise foi realizada no Laboratório de Análise de Solo da Estação Experimental de São Joaquim, vinculado à Rede Oficial de Laboratórios de Análise de Solos dos Estados do Rio Grande do Sul e de Santa Catarina.

O pomar foi instalado no inverno de 2010, em área previamente corrigida de acordo com análise química do solo e recomendações do manual de adubação e calagem para a cultura (Manual..., 2004). Para a correção da acidez do solo, foram incorporados 9,0 Mg de calcário calcítico $+3,0 \mathrm{Mg}$ de calcário dolomítico, para elevar o pH do solo a 6,0 . A correção dos teores de fósforo e potássio foi realizada por meio da incorporação de $243 \mathrm{~kg} \mathrm{ha}^{-1}$ de superfosfato triplo $\left(41 \%\right.$ de $\left.\mathrm{P}_{2} \mathrm{O}_{5}\right)$ e de $42 \mathrm{~kg} \mathrm{ha}^{-1}$ de cloreto de potássio $\left(60 \%\right.$ de $\left.\mathrm{K}_{2} \mathrm{O}\right)$, respectivamente. Foram utilizadas mudas das cultivares Imperial Gala e Mishima Fuji com haste simples, ou seja, sem ramificações laterais. O espaçamento utilizado foi de $1,5 \mathrm{~m}$ entre plantas e 
4,5 m entre linhas (população final de 1.482 plantas por hectare). As plantas foram conduzidas no sistema líder central, em estrutura de sustentação composta de arame, com três fios em cada linha de plantio, e foram mantidas a aproximadamente $3,5 \mathrm{~m}$ de altura. As cultivares se complementam como fornecedoras de
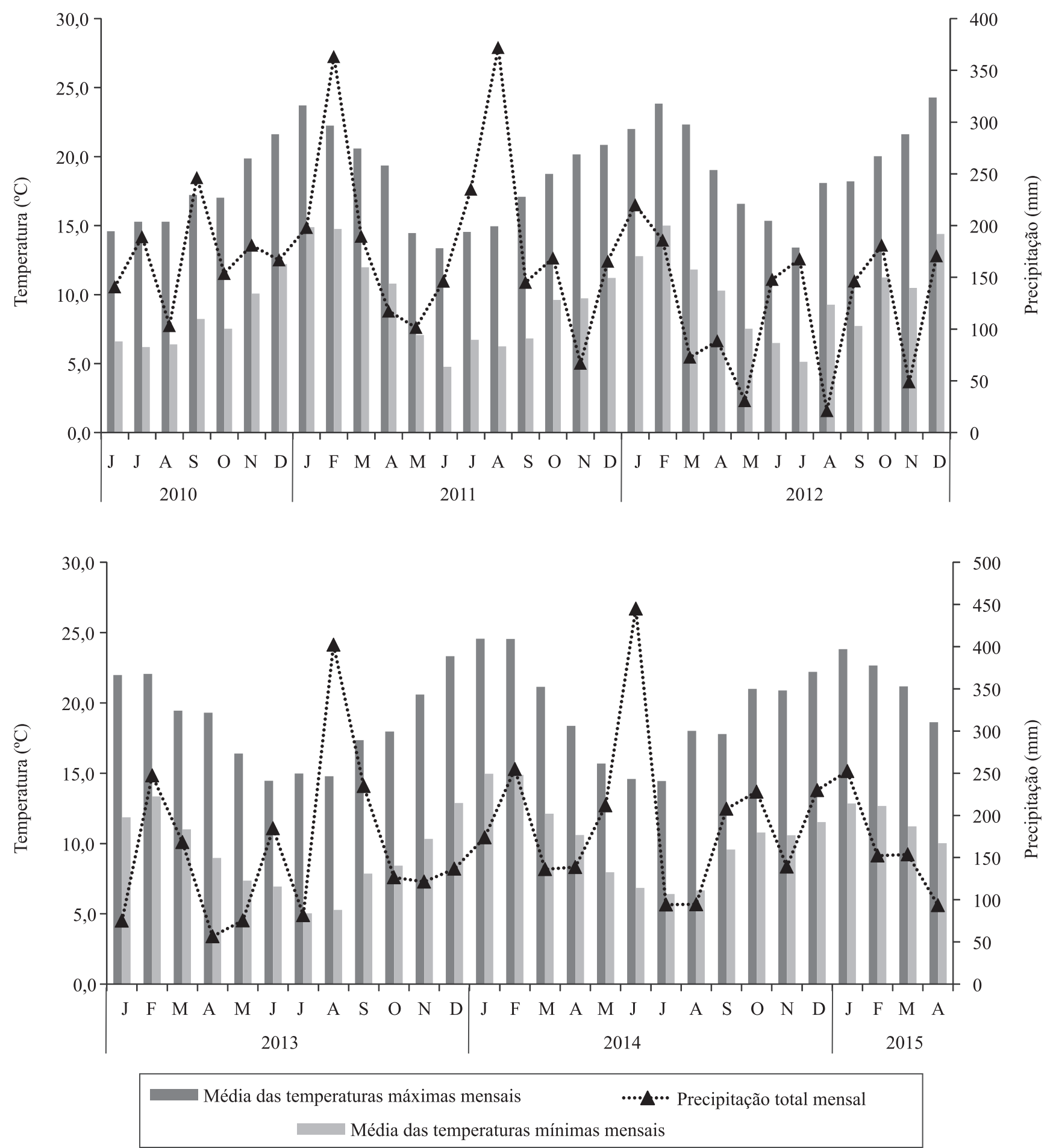

Figura 1. Precipitação mensal e média das temperaturas máximas e minimas mensais observadas no campo experimental localizado em São Joaquim, no Estado de Santa Catarina. Dados obtidos da estação meteorológica localizada na Estação Experimental de São Joaquim da Empresa de Pesquisa Agropecuária e Extensão Rural de Santa Catarina. 
pólen. O manejo do pomar foi realizado de acordo com recomendações do sistema de produção da macieira (A cultura..., 2006). Já a adubação de produção foi realizada anualmente com aplicação de 150 e $100 \mathrm{~g}$ por planta de superfosfato triplo e cloreto de potássio, para suprir as necessidades de fósforo e potássio, respectivamente, no início da brotação. A adubação nitrogenada foi realizada pela aplicação de $100 \mathrm{~g}$ de ureia ( $45 \%$ de nitrogênio) por planta e parcelada em duas vezes: $50 \mathrm{~g}$ no início da brotação e $50 \mathrm{~g}$ na queda de pétalas. Os nutrientes foram distribuídos uniformemente na área correspondente à projeção da copa.

Os tratamentos consistiram em nove porta-enxertos: CG.969, CG.874, CG.210, CG.56, CG.008 e CG.24 da série americana Cornell-Geneva (CG); JM.2 e JM.7 da série japonesa Japan, Morioka (JM); e, como testemunha, a combinação do porta-enxerto Marubakaido com filtro de M.9 (Marubakaido/M.9), este com $15 \mathrm{~cm}$ de comprimento. Cada cultivar foi considerada como um experimento separado, pois não houve casualização das cultivares nos blocos, apenas dos porta-enxertos por cultivar. Utilizou-se, para os dois experimentos o delineamento experimental de blocos ao acaso, com quatro repetições (parcelas). Cada parcela foi constituída por cinco plantas, mas apenas as três plantas centrais foram utilizadas nas avaliações, tendo-se deixado uma planta em cada extremidade como bordadura.

A colheita foi realizada quando os frutos estavam no ponto de maturação comercial, de acordo com o índice iodo-amido (4-5), a firmeza de polpa (80-90 Newton) e os sólidos solúveis (11-12 ${ }^{\circ}$ Brix) (A cultura..., 2006). Por ocasião da colheita, todos os frutos das plantas avaliadas foram colhidos, contados e pesados. Com esses dados, foram calculadas: a produção por planta $(\mathrm{kg})$; a massa de fruto $(\mathrm{g})$, obtida pela relação entre a produção por planta e o número de frutos; e a produtividade $\left(\mathrm{Mg} \mathrm{ha}^{-1}\right)$, obtida pela multiplicação da produção por planta pelo número de plantas por hectare. Ao final de cada ciclo vegetativo, a circunferência do tronco $(\mathrm{cm})$ foi mensurada a $5,0 \mathrm{~cm}$ acima do ponto de enxertia e expressa como área da seção transversal do tronco, calculada como descrito por Pasa et al. (2012).

No momento da colheita, na safra de 2015, foram coletadas amostras de 15 frutos por repetição para as análises qualitativas. Para a análise da firmeza de polpa $(\mathrm{N})$, foram removidas secções de aproximadamente
$1,5 \mathrm{~cm}$ de diâmetro na região equatorial dos frutos, em dois lados opostos, tendo-se utilizado o aparelho "Fruit Texture Analyzer" (Güss Manufacturing, Strand, África do Sul) com ponteira de $11 \mathrm{~mm}$. Em seguida, uma amostra composta foi triturada para obter o suco, o qual foi extraído com pipeta e levado a refratômetro digital, modelo PR-32 (Atago Co., Ltd., Tóquio, Japão), para determinar os sólidos solúveis, expressos como ${ }^{\circ}$ Brix. O índice iodo-amido foi determinado pela reação do amido com uma solução com $12 \mathrm{~g}$ de iodo metálico e $24 \mathrm{~g}$ de iodeto de potássio em 1,0 L de água destilada. Os frutos de cada repetição foram cortados individualmente na região equatorial, e a solução de iodo foi aplicada em uma das metades. A reação do amido da polpa dos frutos com o iodo produziu um padrão de cor para cada fruto, o qual foi comparado com a Tabela desenvolvida pela Empresa de Pesquisa Agropecuária e Extensão Rural de Santa Catarina (A cultura..., 2006), em que o estádio 1 corresponde a frutos muito verdes, e o 9 , a frutos muito maduros.

As análises estatísticas foram realizadas com uso do programa R (R Foundation for Statistical Computing, Viena, Áustria), com o pacote ExpDes (Ferreira et al., 2013). A análise de variância foi realizada pelo teste $\mathrm{F}$, e, quando este foi significativo, os dados foram submetidos à comparação de médias pelo teste de Scott-Knott, a 5\% de probabilidade.

\section{Resultados e Discussão}

A produtividade diferiu significativamente em função dos porta-enxertos em todos os anos de avaliação, para ambas as cultivares, exceto em 2012, para Mishima Fuji (Tabela 1). A produtividade em 2012 foi maior para 'Imperial Gala' com os porta-enxertos JM.2, CG.008 e CG.874, e, em 2013, com Marubakaido/M.9, JM.7, CG.008, CG.874 e CG.56. Efeito similar foi verificado para 'Mishima Fuji', porém com o porta-enxerto CG.210. Em 2014, as maiores produtividades foram obtidas para Marubakaido/M.9 com 'Imperial Gala', e Marubakaido/M.9, CG.008 e CG.56 com 'Mishima Fuji'. Já em 2015, os porta-enxertos JM.7, CG.24 e CG.969 com 'Imperial Gala' foram os menos produtivos, e CG.24 e CG.969 foram ainda menos produtivos com 'Mishima Fuji'. Em relação à produtividade acumulada, para ambas as cultivares, os maiores valores foram observados com Marubakaido/M.9, JM.2, CG.008, 
CG.874, CG.210 e CG.56, e os menores com CG.24 e CG.969.

A produtividade de todos os porta-enxertos da série CG (exceto CG.969 e CG.24) e do JM.2 foi similar à da combinação Marubakaido/M.9. Russo et al. (2007), em estudo com a cultivar Golden Delicious, obtiveram maior produtividade com os porta-enxertos CG.874 e CG.210. Por sua vez, Cline et al. (2010) relataram maior produtividade com uso do porta-enxerto CG.008 nas cultivares Jonagold, McIntosh e Novaspy. A maior produtividade é uma característica dos porta-enxertos dessa série quando comparados aos porta-enxertos ananizantes e semiananizantes mundialmente utilizados, como M.9, M.26 e M.27 (Fazio et al., 2013). Entretanto, à exceção do presente estudo, até o momento, não foram localizados relatos de trabalhos que tenham comparado o desempenho dos porta-enxertos da série CG com o do Marubakaido/M.9. O maior ângulo de inserção dos ramos, tradicionalmente obtido pela prática do arqueamento, está relacionado à maior formação de gemas floríferas em macieiras 'Gala' e 'Fuji' (Zhang et al., 2015) e pode ser um dos motivos do maior potencial produtivo induzido pelos porta-enxertos da série $\mathrm{CG}$, os quais induzem maior ângulo de abertura dos ramos laterais nas cultivares-copa (Fazio \& Robinson, 2008).

Os resultados de produção por planta, para ambas as cultivares, foram semelhantes aos de produtividade (Tabela 2). A única diferença ocorreu na produção acumulada de 'Imperial Gala', a qual foi maior com Marubakaido/M.9, JM.2 e CG.008; intermediária com CG.874, CG.210 e CG.56; e menor com JM.7, CG.24 e CG.969.

A variável número de frutos por planta apresentou diferença significativa em ambas as cultivares, em todos os anos de avaliação (Tabela 3). Em 2012, os maiores valores foram observados para 'Imperial Gala' com JM.2, CG.008 e CG.874, e para 'Mishima Fuji' com Marubakaido/M.9, JM.7 e CG.210. Em 2013, o maior número de frutos por planta foi constatado em 'Imperial Gala' com JM.2, CG.008 e CG.56, e em 'Mishima Fuji' com Marubakaido/M.9, CG.008, CG.874, CG.210 e CG.56. Em 2014, Marubakaido/M.9 foi superior aos

Tabela 1. Produtividade das cultivares de macieira Imperial Gala e Mishima Fuji com diferentes porta-enxertos, de 2012 a 2015 e acumulada ${ }^{(1)}$.

\begin{tabular}{|c|c|c|c|c|c|}
\hline \multirow[t]{2}{*}{ Porta-enxerto } & \multicolumn{5}{|c|}{ Produtividade $\left(\mathrm{Mg} \mathrm{ha}^{-1}\right)$} \\
\hline & 2012 & 2013 & 2014 & 2015 & Acumulada \\
\hline & \multicolumn{5}{|c|}{ 'Imperial Gala' } \\
\hline Marubakaido/M.9 & $5,6 \mathrm{~b}$ & $14,2 \mathrm{a}$ & $41,8 \mathrm{a}$ & $36,0 \mathrm{a}$ & $87,1 \mathrm{a}$ \\
\hline JM.2 & $6,6 a$ & $17,6 \mathrm{a}$ & $27,7 b$ & $36,6 a$ & $88,5 \mathrm{a}$ \\
\hline CG.008 & $9,0 \mathrm{a}$ & $20,9 \mathrm{a}$ & $31,7 b$ & $41,2 \mathrm{a}$ & $102,8 \mathrm{a}$ \\
\hline CG.874 & $6,4 \mathrm{a}$ & $15,9 \mathrm{a}$ & $27,0 \mathrm{~b}$ & $30,5 \mathrm{a}$ & $79,8 \mathrm{a}$ \\
\hline CG.210 & $3,8 b$ & $10,2 b$ & $25,1 b$ & $33,7 \mathrm{a}$ & $75,4 \mathrm{a}$ \\
\hline CG.56 & $5,4 \mathrm{~b}$ & $17,4 \mathrm{a}$ & $26,1 b$ & $28,5 \mathrm{a}$ & $77,4 \mathrm{a}$ \\
\hline JM.7 & $3,5 b$ & $8,7 \mathrm{~b}$ & $21,4 \mathrm{c}$ & $20,3 b$ & $54,0 \mathrm{~b}$ \\
\hline CG.24 & $3,9 b$ & $7,4 \mathrm{~b}$ & $13,3 \mathrm{~d}$ & $13,1 b$ & $33,2 \mathrm{c}$ \\
\hline CG.969 & $4,5 b$ & $7,0 \mathrm{~b}$ & $10,5 \mathrm{~d}$ & $12,2 b$ & $31,6 \mathrm{c}$ \\
\hline \multirow[t]{2}{*}{$\mathrm{p}$} & 0,015 & $<0,001$ & $<0,001$ & $<0,001$ & $<0,001$ \\
\hline & \multicolumn{5}{|c|}{ 'Mishima Fuji' } \\
\hline Marubakaido/M.9 & 6,3 & $18,3 \mathrm{a}$ & $38,0 \mathrm{a}$ & $30,4 \mathrm{a}$ & $93,1 \mathrm{a}$ \\
\hline JM.2 & 6,1 & $12,0 \mathrm{~b}$ & $30,5 \mathrm{a}$ & $32,7 \mathrm{a}$ & $81,3 a$ \\
\hline CG.008 & 4,0 & $19,1 \mathrm{a}$ & $25,1 \mathrm{~b}$ & $38,6 \mathrm{a}$ & $86,9 a$ \\
\hline CG.874 & 3,2 & $16,0 \mathrm{a}$ & $21,9 b$ & $28,4 \mathrm{a}$ & $69,6 a$ \\
\hline CG.210 & 4,3 & $17,7 \mathrm{a}$ & $23,3 b$ & $31,0 \mathrm{a}$ & $76,3 a$ \\
\hline CG.56 & 2,5 & $17,5 \mathrm{a}$ & $29,4 \mathrm{a}$ & $24,0 \mathrm{~b}$ & $73,5 \mathrm{a}$ \\
\hline JM.7 & 4,6 & $12,2 b$ & $16,3 \mathrm{c}$ & $20,3 b$ & $53,3 b$ \\
\hline CG.24 & 4,1 & $9,2 \mathrm{~b}$ & $7,5 \mathrm{~d}$ & $9,6 \mathrm{c}$ & $30,6 c$ \\
\hline CG.969 & 2,4 & $4,5 \mathrm{~b}$ & $6,2 d$ & $6,0 \mathrm{c}$ & $19,2 \mathrm{c}$ \\
\hline $\mathrm{p}$ & 0,085 & 0,005 & $<0,001$ & $<0,001$ & $<0,001$ \\
\hline
\end{tabular}

${ }^{(1)}$ Médias seguidas de letras iguais, nas colunas, não diferem pelo teste de Scott-Knott, a 5\% de probabilidade. 
Tabela 2. Produção por planta das cultivares de macieira Imperial Gala e Mishima Fuji com diferentes porta-enxertos, de 2012 a 2015 e acumulada ${ }^{(1)}$.

\begin{tabular}{lcrrrr}
\hline Porta-enxerto & \multicolumn{5}{c}{ Produção por planta (kg) } \\
\cline { 2 - 6 } & 2012 & \multicolumn{5}{c}{2013} & 2015 & Acumulada \\
\hline \multirow{5}{*}{ Marubakaido/M.9 9} & $3,7 \mathrm{~b}$ & $9,5 \mathrm{a}$ & $28,5 \mathrm{a}$ & $24,3 \mathrm{a}$ & $66,1 \mathrm{a}$ \\
JM.2 & $4,5 \mathrm{a}$ & $11,9 \mathrm{a}$ & $18,7 \mathrm{~b}$ & $24,7 \mathrm{a}$ & $59,7 \mathrm{a}$ \\
CG.008 & $6,1 \mathrm{a}$ & $14,1 \mathrm{a}$ & $21,4 \mathrm{~b}$ & $27,8 \mathrm{a}$ & $69,4 \mathrm{a}$ \\
CG.874 & $4,3 \mathrm{a}$ & $10,7 \mathrm{a}$ & $18,2 \mathrm{~b}$ & $20,6 \mathrm{a}$ & $53,8 \mathrm{~b}$ \\
CG.210 & $2,6 \mathrm{~b}$ & $6,9 \mathrm{~b}$ & $16,9 \mathrm{~b}$ & $22,7 \mathrm{a}$ & $49,1 \mathrm{~b}$ \\
CG.56 & $3,7 \mathrm{~b}$ & $11,7 \mathrm{a}$ & $17,6 \mathrm{~b}$ & $19,2 \mathrm{a}$ & $52,2 \mathrm{~b}$ \\
JM.7 & $2,4 \mathrm{~b}$ & $5,9 \mathrm{~b}$ & $14,5 \mathrm{c}$ & $13,7 \mathrm{~b}$ & $36,4 \mathrm{c}$ \\
CG.24 & $2,6 \mathrm{~b}$ & $5,0 \mathrm{c}$ & $8,8 \mathrm{~d}$ & $8,8 \mathrm{~b}$ & $25,2 \mathrm{c}$ \\
CG.969 & $3,1 \mathrm{~b}$ & $4,8 \mathrm{c}$ & $7,1 \mathrm{~d}$ & $8,2 \mathrm{~b}$ & $23,1 \mathrm{c}$ \\
\hline p & 0,014 & $<0,001$ & $<0,001$ & $<0,001$ & $<0,001$ \\
\hline \multicolumn{5}{c}{ 'Mishima Fuji' } \\
Marubakaido/M.9 & 4,3 & $12,4 \mathrm{a}$ & $25,7 \mathrm{a}$ & $20,5 \mathrm{a}$ & $62,8 \mathrm{a}$ \\
JM.2 & 4,1 & $8,1 \mathrm{~b}$ & $20,6 \mathrm{a}$ & $22,1 \mathrm{a}$ & $54,9 \mathrm{a}$ \\
CG.008 & 2,7 & $12,9 \mathrm{a}$ & $17,0 \mathrm{~b}$ & $26,1 \mathrm{a}$ & $58,7 \mathrm{a}$ \\
CG.874 & 2,2 & $10,8 \mathrm{a}$ & $14,8 \mathrm{~b}$ & $19,2 \mathrm{a}$ & $46,9 \mathrm{a}$ \\
CG.210 & 2,9 & $11,9 \mathrm{a}$ & $15,7 \mathrm{~b}$ & $20,9 \mathrm{a}$ & $51,5 \mathrm{a}$ \\
CG.56 & 1,7 & $11,8 \mathrm{a}$ & $19,9 \mathrm{a}$ & $16,2 \mathrm{~b}$ & $49,6 \mathrm{a}$ \\
JM.7 & 3,1 & $8,2 \mathrm{~b}$ & $11,0 \mathrm{c}$ & $13,7 \mathrm{~b}$ & $36,0 \mathrm{~b}$ \\
CG.24 & 2,8 & $6,2 \mathrm{~b}$ & $5,1 \mathrm{~d}$ & $6,5 \mathrm{c}$ & $20,6 \mathrm{c}$ \\
CG.969 & 1,6 & $3,1 \mathrm{~b}$ & $4,2 \mathrm{~d}$ & $4,0 \mathrm{c}$ & $12,9 \mathrm{c}$ \\
\hline p & 0,08 & 0,01 & $<0,001$ & $<0,001$ & $<0,001$ \\
\hline
\end{tabular}

(1)Médias seguidas de letras iguais, nas colunas, não diferem pelo teste de Scott-Knott, a $5 \%$ de probabilidade. demais porta-enxertos. Já em 2015, o menor número de frutos foi observado em JM.7, CG.24 e CG.969 com 'Imperial Gala', e em CG.24 e CG.969 com 'Mishima Fuji'. O número de frutos por planta acumulado, em 'Imperial Gala', foi maior com Marubakaido/M.9, JM.2 e CG.008 e menor com CG.24 e CG.969. Em 'Mishima Fuji', os maiores valores foram observados com Marubakaido/M.9, JM.2, CG.008, CG.874, CG.210 e CG.56.

A produção e o número de frutos por planta apresentaram relação direta com o vigor induzido pelos porta-enxertos, principalmente em 'Imperial Gala', e, provavelmente, resultaram da maior capacidade dos porta-enxertos mais vigorosos em se estabelecer rapidamente. Larsen et al. (1992) verificaram que a produção e o número de frutos por planta ao longo dos anos, em macieiras 'Delicious' e 'Golden Delicious', tende a aumentar nos porta-enxertos vigorosos e a diminuir naqueles menos vigorosos. Quanto à cultivar Mishima Fuji, no presente trabalho, não foi observada essa tendência, pois a produção por planta dos porta-enxertos de vigor intermediário (CG.008, CG.874 e CG.210) não se diferenciou da dos mais vigorosos (Marubakaido/M.9 e JM.2). Denardi et al. (2015) relataram efeito semelhante para a cultivar

Tabela 3. Número de frutos por planta das cultivares de macieira Imperial Gala e Mishima Fuji com diferentes porta-enxertos, de 2012 a 2015 e acumulado ${ }^{(1)}$.

\begin{tabular}{|c|c|c|c|c|c|}
\hline \multirow[t]{2}{*}{ Porta-enxerto } & \multicolumn{5}{|c|}{ Número de frutos por planta } \\
\hline & 2012 & 2013 & 2014 & 2015 & Acumulado \\
\hline & \multicolumn{5}{|c|}{ 'Imperial Gala' } \\
\hline Marubakaido/M.9 & $24,0 \mathrm{~b}$ & $60,0 \mathrm{~b}$ & $208,5 \mathrm{a}$ & $158,4 \mathrm{a}$ & $450,8 \mathrm{a}$ \\
\hline JM.2 & $27,9 a$ & $89,6 \mathrm{a}$ & $132,2 b$ & $158,2 \mathrm{a}$ & $407,8 \mathrm{a}$ \\
\hline CG.008 & $37,6 \mathrm{a}$ & $101,5 \mathrm{a}$ & $149,4 b$ & $164,6 \mathrm{a}$ & $453,1 \mathrm{a}$ \\
\hline CG. 874 & $27,6 \mathrm{a}$ & $64,3 b$ & $128,5 b$ & $123,0 \mathrm{a}$ & $343,4 b$ \\
\hline CG.210 & $17,8 b$ & $46,8 \mathrm{c}$ & $120,4 b$ & $144,9 \mathrm{a}$ & $329,9 b$ \\
\hline CG.56 & $22,8 b$ & $76,1 \mathrm{a}$ & $124,3 b$ & $120,0 \mathrm{a}$ & $343,2 b$ \\
\hline JM.7 & $13,3 b$ & $44,0 \mathrm{c}$ & $101,8 \mathrm{c}$ & $83,2 b$ & $242,4 \mathrm{c}$ \\
\hline CG.24 & $14,7 \mathrm{~b}$ & $33,8 \mathrm{c}$ & $63,3 \mathrm{c}$ & $56,7 \mathrm{~b}$ & $168,5 \mathrm{~d}$ \\
\hline CG.969 & $19,1 b$ & $28,4 \mathrm{c}$ & $49,9 \mathrm{c}$ & $47,7 \mathrm{~b}$ & $145,1 \mathrm{~d}$ \\
\hline \multirow[t]{2}{*}{$\mathrm{p}$} & 0,013 & $<0,001$ & $<0,001$ & $<0,001$ & $<0,001$ \\
\hline & \multicolumn{5}{|c|}{ 'Mishima Fuji' } \\
\hline Marubakaido/M.9 & $22,5 \mathrm{a}$ & $57,3 a$ & $117,9 \mathrm{a}$ & $105,1 \mathrm{a}$ & $302,9 \mathrm{a}$ \\
\hline JM.2 & $20,1 \mathrm{a}$ & $37,8 b$ & $87,6 \mathrm{~b}$ & $99,3 \mathrm{a}$ & $244,7 \mathrm{a}$ \\
\hline CG.008 & $12,9 b$ & $58,5 \mathrm{a}$ & $71,1 \mathrm{~b}$ & $125,9 \mathrm{a}$ & $268,4 \mathrm{a}$ \\
\hline CG. 874 & $10,6 b$ & $49,7 \mathrm{a}$ & $69,8 b$ & $96,8 \mathrm{a}$ & $226,9 a$ \\
\hline CG. 210 & $15,1 \mathrm{a}$ & $49,9 a$ & $72,8 b$ & $102,5 \mathrm{a}$ & $240,2 \mathrm{a}$ \\
\hline CG.56 & $9,1 b$ & $56,8 \mathrm{a}$ & $88,0 \mathrm{~b}$ & $74,2 \mathrm{~b}$ & $228,1 \mathrm{a}$ \\
\hline JM.7 & $14,3 b$ & $37,7 \mathrm{~b}$ & $46,9 \mathrm{c}$ & $63,6 \mathrm{c}$ & $162,5 b$ \\
\hline CG.24 & $13,8 b$ & $30,0 \mathrm{~b}$ & $22,3 \mathrm{c}$ & $30,3 d$ & $96,3 \mathrm{c}$ \\
\hline CG.969 & $7,0 \mathrm{~b}$ & $12,9 \mathrm{~b}$ & $19,7 \mathrm{c}$ & $18,5 \mathrm{~d}$ & $58,1 \mathrm{c}$ \\
\hline $\mathrm{p}$ & 0,041 & 0,004 & $<0,001$ & $<0,001$ & $<0,001$ \\
\hline
\end{tabular}

${ }^{(1)}$ Médias seguidas de letras iguais, nas colunas, não diferem pelo teste de Scott-Knott, a 5\% de probabilidade. 
Mishima Fuji, ao avaliar a produção por planta dos porta-enxertos de vigor intermediário, entre os quais CG.210 foi inclusive superior ao semivigoroso MM.111.

Quanto à variável área da seção transversal do tronco, diferenças significativas foram constatadas em todos os anos de avaliação, em ambas as cultivares (Tabela 4). Na cultivar Imperial Gala, o porta-enxerto Marubakaido/M.9 apresentou a maior área da seção transversal do tronco em todos os anos de avaliação, seguido de JM.7, não tendo diferido deste em 2014. Com relação a 'Mishima Fuji', a maior área da seção transversal do tronco foi obtida em: 2011, 2012 e 2015, com Marubakaido/M.9 e JM.2; em 2013, com Marubakaido/M.9, JM.2 e CG.874; e, em 2014, apenas com CG.969 e CG.24, os quais foram inferiores aos demais porta-enxertos. O maior incremento da área da seção transversal do tronco ocorreu com os porta-enxertos Marubakaido/M.9, JM.2, CG.008 e CG.210, em ambas as cultivares, e com CG.56 e JM.7 em Mishima Fuji.

Os resultados obtidos no presente trabalho foram semelhantes aos descritos por outros autores com os porta-enxertos CG.874, CG.210 (Russo et al., 2007), CG.210 (Denardi et al., 2015) e CG.008 (Cline et al., 2010), os quais apresentaram vigor intermediário com as cultivares Imperial Gala e Mishima Fuji. Os resultados observados com JM.2 (semiananizante) e JM.7 (ananizante) são condizentes com os encontrados por Russo et al. (2007), para a cultivar Gala, e por Soejima et al. (1998) para a macieira 'Fuji'. A existência de diferentes níveis de vigor entre os porta-enxertos estudados é muito importante para o cultivo da macieira. Segundo Wertheim (2002), além da influência do porta-enxerto, o vigor das plantas enxertadas também é determinado pelas condições de solo, clima e cultivar-copa. Dessa forma, porta-enxertos vigorosos podem ser utilizados em solos com fertilidade natural baixa e/ou rasos, enquanto os pouco vigorosos podem ser usados em solos de alta fertilidade, o que permite o equilíbrio vegeto-produtivo da cultivar-copa.

Houve correlação linearpositiva entre a produtividade e o incremento da área da seção transversal do tronco para ambas as cultivares, ou seja, um aumento na área do tronco corresponde a um aumento na produtividade (Figura 2). No entanto, pôde-se observar que, nos

Tabela 4. Área da seção transversal do tronco das cultivares de macieira Imperial Gala e Mishima Fuji com diferentes porta-enxertos, de 2011 a $2015^{(1)}$.

\begin{tabular}{|c|c|c|c|c|c|c|}
\hline \multirow[t]{2}{*}{ Porta-enxerto } & \multicolumn{5}{|c|}{ Área da seção transversal do tronco $\left(\mathrm{cm}^{2}\right)$} & \multirow{2}{*}{$\begin{array}{l}\text { Incremento de } \\
2011 \text { a } 2015(\%)\end{array}$} \\
\hline & 2011 & 2012 & 2013 & 2014 & 2015 & \\
\hline & \multicolumn{6}{|c|}{ 'Imperial Gala' } \\
\hline Marubakaido/M.9 & $13,2 \mathrm{a}$ & $24,8 \mathrm{a}$ & $37,6 \mathrm{a}$ & $44,3 \mathrm{a}$ & $72,9 \mathrm{a}$ & $469,5 \mathrm{a}$ \\
\hline 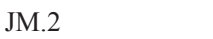 & $10,3 b$ & $18,8 \mathrm{~b}$ & $30,8 b$ & $42,0 \mathrm{a}$ & $59,5 b$ & $491,3 \mathrm{a}$ \\
\hline CG.008 & $7,4 \mathrm{c}$ & $13,4 \mathrm{c}$ & $21,4 \mathrm{c}$ & $30,9 b$ & $39,5 \mathrm{c}$ & $448,8 \mathrm{a}$ \\
\hline CG.874 & $8,9 \mathrm{c}$ & $14,6 \mathrm{c}$ & $20,9 \mathrm{c}$ & $25,5 \mathrm{c}$ & $35,4 \mathrm{c}$ & $301,0 \mathrm{~b}$ \\
\hline CG.210 & $7,1 \mathrm{c}$ & $12,3 \mathrm{c}$ & $20,9 \mathrm{c}$ & $27,8 b$ & $42,6 \mathrm{c}$ & $536,1 \mathrm{a}$ \\
\hline CG.56 & $7,6 \mathrm{c}$ & $11,5 \mathrm{c}$ & $15,5 \mathrm{~d}$ & $19,6 \mathrm{c}$ & $32,1 \mathrm{c}$ & $321,9 b$ \\
\hline JM.7 & $6,4 \mathrm{c}$ & $10,9 \mathrm{c}$ & $16,0 \mathrm{~d}$ & $20,1 \mathrm{c}$ & $25,4 d$ & $295,3 b$ \\
\hline CG.969 & $6,1 \mathrm{c}$ & $6,2 d$ & $8,2 \mathrm{e}$ & $9,4 \mathrm{~d}$ & $14,3 \mathrm{e}$ & $153,8 b$ \\
\hline CG.24 & $3,9 d$ & $6,4 d$ & $9,2 \mathrm{e}$ & $10,2 \mathrm{~d}$ & $13,9 \mathrm{e}$ & $257,2 b$ \\
\hline \multirow[t]{2}{*}{$\mathrm{p}$} & $<0,001$ & $<0,001$ & $<0,001$ & $<0,001$ & $<0,001$ & $<0,001$ \\
\hline & \multicolumn{6}{|c|}{ 'Mishima Fuji' } \\
\hline Marubakaido/M.9 & $14,5 \mathrm{a}$ & $23,2 \mathrm{a}$ & $29,0 \mathrm{a}$ & $40,0 \mathrm{a}$ & $55,0 \mathrm{a}$ & $283,7 b$ \\
\hline JM.2 & $12,6 \mathrm{a}$ & $21,5 \mathrm{a}$ & $29,4 a$ & $38,7 \mathrm{a}$ & $60,0 \mathrm{a}$ & $383,6 \mathrm{a}$ \\
\hline CG.008 & $9,5 b$ & $15,7 \mathrm{~b}$ & $23,9 b$ & $33,7 \mathrm{a}$ & $48,7 \mathrm{~b}$ & $409,5 \mathrm{a}$ \\
\hline CG. 874 & $10,5 b$ & $17,9 \mathrm{~b}$ & $25,7 \mathrm{a}$ & $34,8 \mathrm{a}$ & $42,0 \mathrm{~b}$ & $304,1 b$ \\
\hline CG. 210 & $8,9 b$ & $15,2 b$ & $22,4 b$ & $30,8 \mathrm{a}$ & $46,9 b$ & $440,9 \mathrm{a}$ \\
\hline CG.56 & $8,3 b$ & $14,8 \mathrm{~b}$ & $20,8 b$ & $25,0 \mathrm{a}$ & $40,4 b$ & $389,7 \mathrm{a}$ \\
\hline JM.7 & $9,1 b$ & $15,1 \mathrm{~b}$ & $20,5 b$ & $27,6 \mathrm{a}$ & $40,9 b$ & $345,6 \mathrm{a}$ \\
\hline CG.969 & $4,1 \mathrm{c}$ & $6,1 \mathrm{c}$ & $7,0 \mathrm{c}$ & $9,0 \mathrm{~b}$ & $15,0 \mathrm{c}$ & $271,7 \mathrm{~b}$ \\
\hline CG.24 & $4,7 \mathrm{c}$ & $6,4 \mathrm{c}$ & $7,3 \mathrm{c}$ & $9,4 \mathrm{~b}$ & $13,0 \mathrm{c}$ & $185,7 \mathrm{~b}$ \\
\hline $\mathrm{p}$ & $<0,001$ & $<0,001$ & $<0,001$ & $<0,001$ & $<0,001$ & 0,003 \\
\hline
\end{tabular}

${ }^{(1)}$ Médias seguidas de letras iguais, nas colunas, não diferem pelo teste de Scott-Knott, a 5\% de probabilidade. 
porta-enxertos CG.24 e CG.969, não houve aumento significativo da produtividade de acordo com o aumento da área da seção transversal do tronco. Esse resultado pode ser explicado como efeito da baixa compatibilidade dos porta-enxertos CG.24 e CG.969 com as cultivares-copa estudadas, caracterizada pelo crescimento exagerado da região acima do ponto de enxertia.

O porta-enxerto CG.874 mostrou-se bastante promissor, pois, além de ter apresentado menor incremento da área da seção transversal do tronco, quando comparado a Marubakaido/M.9, em ambas as cultivares, manteve produtividades similares. $\mathrm{O}$ aumento da densidade de plantio é um dos fatores mais importantes para o aumento da produtividade de pomares de macieira (Petri et al., 2011), mas depende de técnicas de manejo para controlar o tamanho das plantas (Pasa \& Einhorn, 2014). Desse modo, o
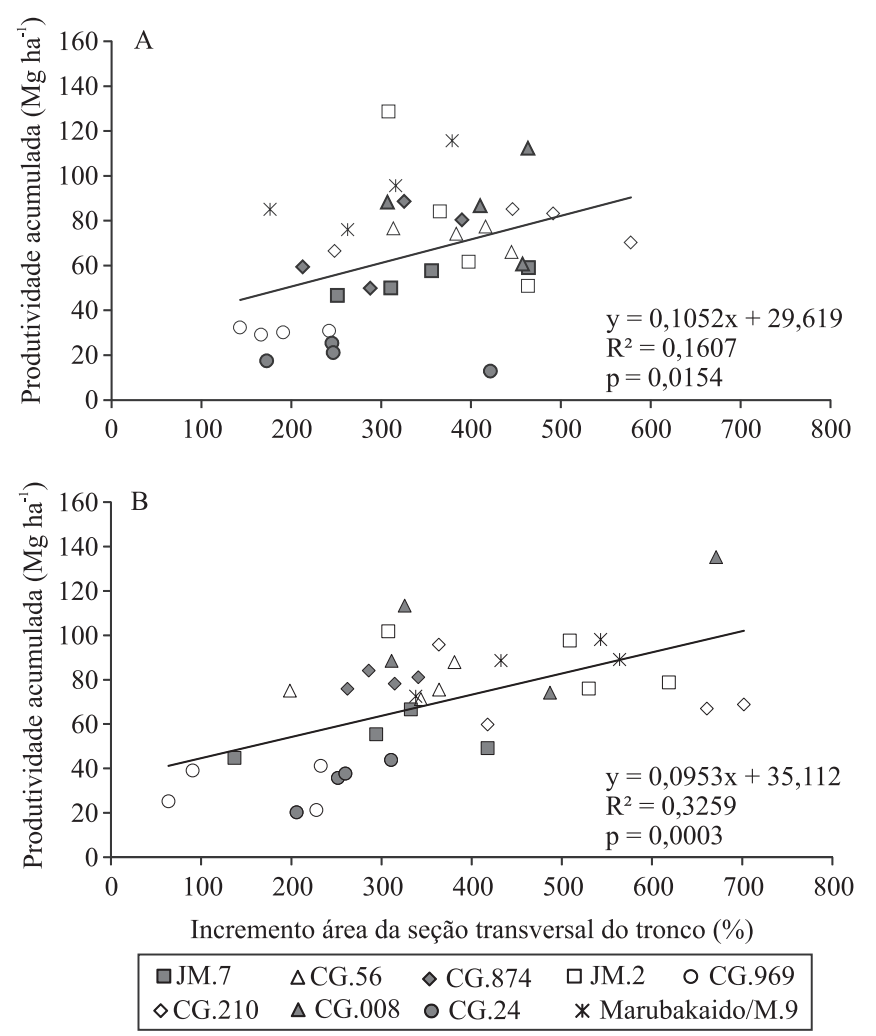

Figura 2. Análise de regressão entre as variáveis produtividade acumulada e área da seção transversal do tronco das cultivares de macieira Mishima Fuji (A) e Imperial Gala (B). porta-enxerto CG.874 apresenta grande potencial para adensamento de pomares.

A massa média de frutos diferiu entre os porta-enxertos apenas em 2012, em 'Imperial Gala', e, em 2014, em 'Mishima Fuji' (Tabela 5). Em 2012, em 'Imperial Gala', a menor massa de fruto foi observada com Marubakaido/M.9, CG.874 e CG.210. Já, em 2014, em 'Mishima Fuji', os frutos das plantas enxertadas em Marubakaido/M.9, CG.874, CG.210 e CG.969 foram menores que os das demais. A massa média de fruto foi menor com Marubakaido/M.9; CG.874 e CG.210; e CG.56 e CG.24. O efeito do porta-enxerto sobre a massa de frutos não foi consistente ao longo dos anos e não parece ter sido decorrente das diferenças no número de frutos (Tabela 3). Esse resultado é comparável aos encontrados por Russo et al. (2007), para as cultivares Golden Delicious, Gala e Honeycrisp, e por Al-Hinai \& Roper (2004), para macieiras 'Gala', os quais observaram pouca influência do porta-enxerto sobre a massa de fruto.

Tabela 5. Massa de fruto das cultivares de macieira Imperial Gala e Mishima Fuji com diferentes porta-enxertos, de 2012 a 2015 e média ${ }^{(1)}$.

\begin{tabular}{|c|c|c|c|c|c|}
\hline \multirow[t]{2}{*}{ Porta-enxerto } & \multicolumn{4}{|c|}{ Massa de fruto $(\mathrm{g})$} & \multirow[t]{2}{*}{ Média } \\
\hline & 2012 & 2013 & 2014 & 2015 & \\
\hline & \multicolumn{5}{|c|}{ 'Imperial Gala' } \\
\hline Marubakaido/M.9 & $156,1 b$ & 165,3 & 135,2 & 155,0 & 153,0 \\
\hline JM.2 & $163,6 \mathrm{a}$ & 133,0 & 142,9 & 156,5 & 149,0 \\
\hline CG.008 & $164,7 \mathrm{a}$ & 139,5 & 142,7 & 169,0 & 154,0 \\
\hline CG.874 & $156,6 \mathrm{~b}$ & 179,3 & 142,7 & 167,5 & 161,5 \\
\hline CG.210 & $144,1 b$ & 145,4 & 142,8 & 156,5 & 147,2 \\
\hline CG.56 & $160,0 \mathrm{a}$ & 155,3 & 142,0 & 162,5 & 155,0 \\
\hline JM.7 & $177,2 \mathrm{a}$ & 132,7 & 145,1 & 165,5 & 155,1 \\
\hline CG.24 & $178,2 \mathrm{a}$ & 149,8 & 141,6 & 155,9 & 156,4 \\
\hline CG.969 & $163,5 \mathrm{a}$ & 171,7 & 143,3 & 172,3 & 162,7 \\
\hline \multirow[t]{2}{*}{$\mathrm{p}$} & 0,029 & 0,520 & 0,890 & 0,354 & 0,585 \\
\hline & \multicolumn{5}{|c|}{ 'Mishima Fuji' } \\
\hline Marubakaido/M.9 & 185,7 & 214,3 & $217,7 \mathrm{~b}$ & 195,5 & $203,3 b$ \\
\hline JM.2 & 206,3 & 207,8 & $234,1 \mathrm{a}$ & 221,5 & $217,4 \mathrm{a}$ \\
\hline CG.008 & 211,5 & 217,8 & $241,7 \mathrm{a}$ & 207,0 & $219,5 \mathrm{a}$ \\
\hline CG.874 & 196,1 & 218,1 & $212,4 b$ & 198,0 & $206,1 b$ \\
\hline CG.210 & 191,3 & 245,0 & $213,7 b$ & 205,0 & $213,7 b$ \\
\hline CG.56 & 187,6 & 208,6 & $225,6 a$ & 217,0 & $209,7 b$ \\
\hline JM.7 & 216,1 & 218,5 & $234,6 a$ & 215,5 & $221,2 \mathrm{a}$ \\
\hline CG.24 & 204,7 & 203,0 & $228,7 \mathrm{a}$ & 215,2 & $212,9 b$ \\
\hline CG.969 & 223,9 & 244,2 & $214,5 b$ & 222,2 & $226,2 \mathrm{a}$ \\
\hline $\mathrm{p}$ & 0,206 & 0,520 & 0,042 & 0,066 & 0,027 \\
\hline
\end{tabular}

${ }^{(1)}$ Médias seguidas de letras iguais, nas colunas, não diferem pelo teste de Scott-Knott, a 5\% de probabilidade. 
Os parâmetros de qualidade dos frutos, com exceção do índice de iodo-amido na cultivar Mishima Fuji, diferiram significativamente entre os porta-enxertos (Tabela 6). A firmeza de polpa foi maior em 'Imperial Gala' com JM.2, bem como em 'Mishima Fuji' com CG.210, CG.56, JM.2, CG.969 e CG.24. Os sólidos solúveis, em 'Imperial Gala', foram maiores com Marubakaido/M.9, CG.210, JM.2, CG.969 e CG.24, e, em Mishima Fuji, com JM.2, CG.969 e CG.24. $\mathrm{O}$ índice iodo-amido diferiu apenas em 'Imperial Gala', em que JM.7, CG.56 e CG.969 apresentaram os maiores valores.

O teor de sólidos solúveis foi o único parâmetro que apresentou diferenças consistentes entre os porta-enxertos em ambas as cultivares; de modo geral, os maiores valores foram observados nos porta-enxertos menos vigorosos. A melhor interceptação luminosa de porta-enxertos menos vigorosos é uma possível explicação para esse resultado, pois a luz afeta a qualidade dos frutos, o que inclui o teor de sólidos solúveis

Tabela 6. Firmeza de polpa, sólidos solúveis e índice iodo-amido dos frutos das cultivares de macieira Imperial Gala e Mishima Fuji com diferentes porta-enxertos, em 2015(1).

\begin{tabular}{|c|c|c|c|}
\hline Porta-enxerto & $\begin{array}{l}\text { Firmeza de polpa } \\
(\mathrm{N})\end{array}$ & $\begin{array}{c}\text { Sólidos solúveis } \\
\left({ }^{\circ} \text { Brix }\right)\end{array}$ & $\begin{array}{c}\text { Índice } \\
\text { iodo-amido }\end{array}$ \\
\hline & \multicolumn{3}{|c|}{ 'Imperial Gala' } \\
\hline Marubakaido/M.9 & $73,0 \mathrm{~b}$ & $10.4 b$ & $7,0 \mathrm{~b}$ \\
\hline JM.2 & $73,6 b$ & $10,5 b$ & $7,4 \mathrm{a}$ \\
\hline CG.008 & $75,9 b$ & $10,4 b$ & $6,9 b$ \\
\hline CG.874 & $74,7 b$ & $11,0 \mathrm{~b}$ & $6,7 b$ \\
\hline CG.210 & $74,0 \mathrm{~b}$ & $11,8 \mathrm{a}$ & $6,9 b$ \\
\hline CG.56 & $71,7 \mathrm{~b}$ & $10,6 b$ & $7,4 \mathrm{a}$ \\
\hline JM.7 & $79,7 \mathrm{a}$ & $11,9 \mathrm{a}$ & $6,9 b$ \\
\hline CG.969 & $73,5 b$ & $12,3 \mathrm{a}$ & $7,6 a$ \\
\hline CG.24 & $73,6 \mathrm{~b}$ & $11,3 \mathrm{a}$ & $6,8 \mathrm{~b}$ \\
\hline \multirow[t]{2}{*}{$\mathrm{p}$} & $<0,001$ & $<0,001$ & 0,006 \\
\hline & \multicolumn{3}{|c|}{ 'Mishima Fuji' } \\
\hline Marubakaido/M.9 & $72,0 \mathrm{~b}$ & $13,2 \mathrm{~b}$ & 7,0 \\
\hline JM.2 & $73,1 b$ & $13,4 b$ & 6,6 \\
\hline CG.008 & $74,8 \mathrm{~b}$ & $12,9 \mathrm{~b}$ & 6,7 \\
\hline CG.874 & $75,2 b$ & $13,2 b$ & 6,9 \\
\hline CG.210 & $76,5 \mathrm{a}$ & $13,1 b$ & 6,7 \\
\hline CG.56 & $78,5 \mathrm{a}$ & $13,9 b$ & 7,1 \\
\hline JM.7 & $77,7 \mathrm{a}$ & $14,3 \mathrm{a}$ & 7,0 \\
\hline CG.969 & $79,9 \mathrm{a}$ & $15,3 \mathrm{a}$ & 6,8 \\
\hline CG.24 & $80,1 \mathrm{a}$ & $14,6 \mathrm{a}$ & 6,7 \\
\hline $\mathrm{p}$ & 0,001 & $<0,001$ & 0,830 \\
\hline
\end{tabular}

${ }^{(1)}$ Médias seguidas de letras iguais, nas colunas, não diferem pelo teste de Scott-Knott, a 5\% de probabilidade.
(Robinson et al., 1983). Além disso, porta-enxertos menos vigorosos apresentam menor volume de raízes em razão da redução no transporte de auxinas da copa para as raízes, o que limita seu crescimento (Hooijdonk et al., 2011). Isso resulta na redução de translocação de água para os frutos no momento da maturação, o que aumenta a concentração de sólidos solúveis.

\section{Conclusões}

1. As cultivares de macieira Imperial Gala e Mishima Fuji são mais produtivas quando enxertadas em Marubakaido/M.9, JM.2, CG.008, CG.874, CG.210 e CG.56.

2. Os porta-enxertos CG.008, CG.874, CG.210 e CG.56 reduzem a área da seção transversal do tronco de ambas as cultivares estudadas, mas sem reduzir a produtividade.

3. O teor de sólidos solúveis dos frutos das cultivares Imperial Gala e Mishima Fuji é maior nos porta-enxertos menos vigorosos.

\section{Referências}

AL-HINAI, Y.K.; ROPER, T.R. Rootstock effects on growth and quality of 'Gala' apples. HortScience, v.39, p.1231-1233, 2004.

BENEZ, M.C. Dados e informações biofísicas da Unidade de Planejamento Regional Planalto Sul Catarinense - UPR 3. In: DUFLOTH, J.H.; CORTINA, N.; VEIGA, M.; MIOR, L.C. (Ed.). Estudos básicos regionais de Santa Catarina. Florianópolis: Epagri, 2005. 76p.

CLINE，J.A.; NORTON，D.; EMBREE，C.G.; PRIVÉ，J.-P. Performance of Jonagold, McIntosh and Novaspy on three new semi-dwarf apple rootstocks in eastern Canada. Canadian Journal of Plant Science, v.90, p.877-883, 2010. DOI: 10.4141/cjps09186.

A CULTURA da macieira. Florianópolis: Epagri, 2006. 743p.

DENARDI, F.; KVITSCHAL, M.V.; BASSO, C.; BONETI, J.I. da S.; KATSURAYAMA, Y. Desempenho agronômico de porta-enxertos de macieira da série americana 'Geneva ${ }^{\mathbb{B} 1}$ no Sul do Brasil. Revista Brasileira de Fruticultura, v.37, p.104-111, 2015. DOI: 10.1590/0100-2945-438/14.

DI VAIO, C.; CIRILLO, C.; BUCCHERI, M.; LIMONGELLI, F. Effect of interstock (M.9 and M.27) on vegetative growth and yield of apple trees (cv "Annurca"). Scientia Horticulturae, v.119, p.270-274, 2009. DOI: 10.1016/j.scienta.2008.08.019.

FACHINELLO, J.C.; PASA, M. da S.; SCHMITZ, J.D.; BETEMPS, D.L. Situação e perspectivas da fruticultura de clima temperado no Brasil. Revista Brasileira de Fruticultura, v.33, p.109-120, 2011. DOI: 10.1590/S0100-29452011000500014.

FAZIO， G.; ALDWINCKLE, H.; ROBINSON, T. Unique characteristics of Geneva $^{\circledR}$ apple rootstocks. New York Fruit Quarterly, v.21, p.25-28, 2013. 
FAZIO, G.; ROBINSON, T. Modification of nursery tree architecture with apple rootstocks: a breeding perspective. New York Fruit Quarterly, v.16, p.13-16, 2008.

FERREIRA, E.B.; CAVALCANTI, P.P.; NOGUEIRA, D.A. ExpDes: experimental designs package. Version 1.1.2. Vienna: R Foundation for Statistical Computing, 2013.

GJAMOVSKI, V.; KIPRIJANOVSKI, M. Influence of nine dwarfing apple rootstocks on vigour and productivity of apple cultivar 'Granny Smith'. Scientia Horticulturae, v.129, p.742-746, 2011. DOI: 10.1016/j.scienta.2011.05.032.

HAMPSON, C.R.; QUAMME, H.A.; BROWNLEE, R.T. Canopy growth, yield, and fruit quality of 'Royal Gala' apple trees grown for eight years in five tree training systems. HortScience, v.37, p.627-631, 2002.

HOOIJDONK, B. van; WOOLLEY, D.; WARRINGTON, I. Rootstocks modify scion architecture, endogenous hormones, and root growth of newly grafted 'Royal Gala' apple trees. Journal of the American Society for Horticultural Science, v.136, p.93-102, 2011.

INSTITUTO BRASILEIRO DE GEOGRAFIA E ESTATÍSTICA. Sistema IBGE de Recuperação Automática - SIDRA. Quantidade produzida e rendimento médio de maçã. 2014. Disponível em: $<$ http://www.sidra.ibge.gov.br/>. Acesso em: 1 jun. 2015.

KREUZ, C.L.; SOUZA, A.; PETRI, J.L. Impacto da intensificação da densidade de plantio na rentabilidade em duas cultivares de macieira em Fraiburgo-SC. Revista Brasileira de Fruticultura, v.28, p.240-243, 2006. DOI: 10.1590/ S0100-29452006000200019.

LARSEN, F.E.; HIGGINS, S.S.; DOLPH, C.A. Rootstock influence over 25 years on yield, yield efficiency and tree growth of cultivars 'Delicious' and 'Golden Delicious' apple (Malus domestica Borkh.). Scientia Horticulturae, v.49, p.63-70, 1992. DOI: 10.1016/0304-4238(92)90143-Z.

MANUAL de adubação e calagem para os Estados do Rio Grande do Sul e Santa Catarina. 10.ed. Porto Alegre: Sociedade Brasileira de Ciência do Solo, Núcleo Regional Sul, Comissão de Química e Fertilidade do Solo, 2004. 400p.

PASA, M.S.; EINHORN, T.C. Heading cuts and prohexadione-calcium affect the growth and development of 'd'Anjou' pear shoots in a high-density orchard. Scientia
Horticulturae, v.168, p.267-271, 2014. DOI: 10.1016/j. scienta.2014.02.003.

PASA, M.S.; FACHINELLO, J.C.; SCHMITZ, J.D.; SOUZA, A.L.K. de; FRANCESCHI, E. Desenvolvimento, produtividade e qualidade de peras sobre porta-enxertos de marmeleiro e Pyrus calleryana. Revista Brasileira de Fruticultura, v.34, p.873-880, 2012. DOI: $10.1590 / \mathrm{S} 0100-29452012000300029$.

PETRI, J.L.; LEITE, G.B.; COUTO, M.; FRANCESCATTO, P. Avanços na cultura da macieira no Brasil. Revista Brasileira de Fruticultura, v.33, p.48-56, 2011. DOI: 10.1590/ S0100-29452011000500007.

ROBINSON, T. Advances in apple culture worldwide. Revista Brasileira de Fruticultura, v.33, p.37-47, 2011. DOI: 10.1590/ S0100-29452011000500006.

ROBINSON, T.; SEELEY, E.J.; BARRITT, B.H. Effect of light environment and spur age on 'Delicious' apple fruit size and quality. Journal of the American Society for Horticultural Science, v.108, p.855-861, 1983.

RUSSO, N.L.; ROBINSON, T.; FAZIO, G.; ALDWINCKLE, H.S. Field evaluation of 64 apple rootstocks for orchard performance and fire blight resistance. HortScience, v.42, p.1517-1525, 2007.

SANTOS, H.G. dos; JACOMINE, P.K.T.; ANJOS, L.H.C. dos; OLIVEIRA, V.A. de; LUMBRERAS, J.F.; COELHO, M.R.; ALMEIDA, J.A. de; CUNHA, T.J.F.; OLIVEIRA, J.B. de. Sistema brasileiro de classificação de solos. 3.ed. Brasília: Embrapa, 2013.353p.

SÍNTESE anual da agricultura de Santa Catarina 2012-2013. Florianópolis: Epagri, 2013. v.34, 178p.

SOEJIMA, J.; BESSHO, H.; TSUCHIYA, S.; KOMORI, S.; ABE, K.; KOTODA, N. Breeding of Fuji apples and performance on JM rootstocks. Compact Fruit Tree, v.31, p.22-24, 1998.

WERTHEIM, S.J. Rootstocks for European pear: a review. Acta Horticulturae, v.596, p.299-309, 2002. DOI: 10.17660/ ActaHortic.2002.596.47.

ZHANG, M.R.; HAN, M.Y.; MA, F.W.; SHU, H.R. Effect of bending on the dynamic changes of endogenous hormones in shoot terminals of 'Fuji' and 'Gala' apple trees. Acta Physiologiae Plantarum, v.37, article 76, 2015. DOI: 10.1007/s11738-015-1813-z.

Recebido em 22 de julho de 2015 e aprovado em 4 de novembro de 2015 